Rev. Elet. em Gestão, Educação e Tecnologia Ambiental （e-ISSN: 2236-1170)

\title{
CAUSAS AMBIENTAIS PARA SÍNDROME DE BURNOUT EM UTI NEONATAL
}

\author{
Leonela Matos ${ }^{1}$, Rodrigo Loreto Peres ${ }^{2}$, Ana Maria Rodrigues da Silva ${ }^{3}$, \\ Juciany Souza Pires ${ }^{4}$, Larissa Lara Lyrio Costa ${ }^{5}$, Danielle da Silva Neves ${ }^{6}$, \\ Rodney Alves Barbosa ${ }^{7}$, Kátia de Fátima Vilela ${ }^{8}$ \\ ${ }^{1}$ leonella_mattos@hotmail.com, ${ }^{2}$ rodrigo.loreto@gmail.com, ${ }^{3}$ nanamel_37@hotmail.com, ${ }^{4}$ \\ juciany.pires@ffassis.edu.br, ${ }^{5}$ larissa.costa@ffassis.edu.br, ${ }^{6}$ dannielly_ferraz@hotmail.com, ${ }^{7}$ \\ rodneyabarbosa@yahoo.com.br, ${ }^{8}$ katia.vilela@ffassis.edu.br
}

\begin{abstract}
RESUMO
O presente estudo tem por finalidade Identificar características comportamentais da Síndrome de Burnout na equipe multiprofissional da UTI neonatal do Hospital Santa Rita no município de Teixeira de Freitas-BA. Nessa perspectiva objetivou-se descrever a influência do ambiente de trabalho nas Unidades de Terapia Intensiva, bem como identificar os fatores laborais que afetam o psicofísico e a produtividade do profissional nessas Unidades. Por se tratar de uma doença característica do meio laboral, que se dá em resposta à cronificação do estresse ocupacional trazendo consigo consequências negativas em nível individual, profissional, familiar e social o tema foi escolhido para alertar sobre a relevância de se encontrar meios para preservar a saúde mental dos profissionais de saúde que têm em sua essência o cuidado. 0 método utilizado foi o descritivo, com abordagem quantitativa e desenvolvido por meio de referências teóricas e pesquisa de campo com 16 profissionais que se propuseram a participar deste estudo. Os instrumentos utilizados nessa pesquisa foram um questionário investigativo com fatores predisponentes pessoais de risco para a Síndrome de Burnout, juntamente com o MBI (Maslach Burnout Inventory) Inventário em Burnout de Maslach, esse questionário é utilizado para estimar a síndrome de Burnout englobando os três aspectos: exaustão emocional, despersonalização e envolvimento pessoal no trabalho. A pesquisa foi realizada com a participação de 16 profissionais que integram a equipe multidisciplinar da UTI neonatal. Como resultado percebe-se que $63 \%$ dos entrevistados se encontram em fase inicial da doença, com nível de estresse moderado, um dado relevante que merece atenção.
\end{abstract}

Palavras chave: Burnout, UTI neonatal, estresse ocupacional, saúde mental.

\section{ABSTRACT}

The present study aims to identify behavioral characteristics of burnout syndrome in the neonatal ICU multidisciplinary team of the Hospital Santa Rita in the city of Teixeira de Freitas, Bahia. From this perspective aimed to describe the influence of work environment in intensive care units, and identify the factors that affect the psychophysical labor and worker productivity in these units. Because it is a disease characteristic of the workplace, which has been in response to the chronicity of occupational stress bringing a negative impact on an individual level, professional, family and social theme was chosen to raise awareness of the importance of finding ways to preserve mental health professionals who have health care at its core. The method used was descriptive, quantitative and references developed through theoretical and field research with 16 professionals who intended to participate in this study. The instruments used in this study were an investigative questionnaire with personal risk factors predisposing to burnout syndrome, along with the $\mathrm{MBI}$ (Maslach Burnout Inventory) Maslach Burnout Inventory in this questionnaire is used to estimate the Burnout syndrome encompassing the three aspects : emotional exhaustion, depersonalization and personal involvement in the work. The survey was conducted with the participation of 16 professionals within the multidisciplinary team from the neonatal ICU. As a result it is observed that $63 \%$ of respondents 


\section{Rev. Elet. em Gestão, Educação e Tecnologia Ambiental (e-ISSN: 2236-1170)}

are in early stages of the disease, with moderate levels of stress, an important finding that deserves attention.

Keywords: Burnout, neonatal ICU, occupational stress, mental health

\section{INTRODUÇÃO}

Com o desenvolvimento social e tecnológico do mundo moderno, podemos perceber que ocorreram mudanças que trouxeram benefícios, mas em troca desse desenvolvimento acelerado, como conseqüência, desenvolveram-se distúrbios psíquicos na população (JODAS e HADDAD 2009; OLIVEIRA, TRISTÃO, NEIVA 2006).

Esses distúrbios fazem com que a mente sofra influências ambientais e com isso agindo de forma errática e desorganizada sobre o corpo. $O$ estilo de vida moderno transformaram as influencias em doenças psicossomáticas desestabilizando as inter-relações no ambiente de trabalho como conseqüência, principalmente, do estresse. Outro aliado de grande participação nas doenças psicossomáticas é a carga excessiva de trabalho decorrente dos aspectos organizacionais e administrativos aos quais os funcionários estão atrelados e continuamente se sentindo obrigados a obedecer acumulando frustrações diárias muitas vezes por anos.

Segundo Filgueiras e Hippert 2002 apud.OLIVEIRA TRISTÃO e NEIVA (2006) o estresse tem como efeitos nos indivíduos, destacando-se no físicos como: dores de cabeça, fadiga, insônia, alterações intestinais, palpitações, náuseas, tremores, extremidades frias, resfriados constantes, e entre os psíquicos: diminuição da concentração e memória, indecisões, confusões, perda de senso de humor, nervosismo, ansiedade, raiva, frustração, irritabilidade, impaciência, e medo.

Os impactos causados pelo estresse ocupacional é um fator preocupante, dentro de uma unidade de terapia intensiva, pois as relações interpessoais entre a equipe e os familiares podem criar reações depressivas, de tristeza, melancolia e pessimismo nos profissionais

A Unidade de Terapia Intensiva (UTI) teve suas origens nas unidades de recuperação pós-anestésica (URPA), onde os pacientes submetidos à procedimentos anestésico-cirúrgicos tinham monitorizadas suas funções vitais até sua recuperação, hoje é o local dentro da instituição de saúde que surgiu da necessidade de oferecer um suporte avançado com atendimento em sistema de vigilância contínua e que admite pacientes graves ou com descompensação de um ou mais sistemas orgânicos, de risco, potencialmente recuperáveis, possuindo monitorização completa (BRASIL, 2011).

A UTI deve estar estruturada no formato para suporte (diagnostico e terapêutico) nos aspectos hemodinâmicos, metabólicos, nutricional, respiratório e de reabilitação, e devem funcionar em espaços individualizados e com equipe própria. Possui normas que determinam sua localização, estrutura física, equipamentos e equipe com particularidade para a UTIN (Unidade de Terapia Intensiva Neonatal)

Uma UTIN tem os mesmos parâmetros estruturais, físicos e de localização de uma Unidade de Terapia Intensiva (UTI). Entretanto nela todos os profissionais de saúde envolvido devem ter como característica uma habilidade especial em cuidados de neonatos.

Ao longo do tempo a assistência neonatal também passou por mudanças significativas ampliando as formas de intervenções aos recém-nascidos (RN) num raciocínio cada vez mais holístico na questão da assistência aos familiares proporcionando uma melhora na qualidade do serviço oferecido saindo de sua lógica e perspectiva racional e somente foco na recuperação do corpo anátomo-fisiológico do RN (GAívA e SCOCHI, 2005).

A UTIN é considerada como local de carga emocional superior aos outros, ambientes hospitalares. O profissional que atua neste lugar está diretamente ligado as emoções de familiares e de toda equipe. $O$ trabalho pode se tornar um fardo, trazendo decepções, medo, agressividade e por fim doenças (SOUZA, 2010). 


\section{Rev. Elet. em Gestão, Educação e Tecnologia Ambiental (e-ISSN: 2236-1170)}

Nestas unidades o fator comum é o estresse. $O$ estresse pode ser medido quantitativamente utilizando marcadores químicos como cortisol (FISHER e col., 2000). Comumente nesses locais não há monitoramento de estresse via marcadores químicos. Nesse sentido a maioria dos profissionais da área de saúde acabam desenvolvendo a Síndrome de Burnout que é o esgotamento físico e mental do profissional quando não identifica e trata seu estresse (SOUZA apud PAFARO; MARTINS, 2002).

Segundo Harrison (apud CARLOTTO, 2002) a síndrome é considerada como um estresse persistente ligado diretamente as situações de trabalho, tendo como conseqüência constante pressão emocional e psicológica, associada ao estreito envolvimento com pessoas por longos períodos.

O presente estudo tem por finalidade Identificar características comportamentais da Síndrome de Burnout na equipe multiprofissional da UTI neonatal do Hospital Santa Rita no município de Teixeira de Freitas-BA. Nessa perspectiva objetivou-se descrever a influência do ambiente de trabalho nas Unidades de Terapia Intensiva, bem como identificar os fatores laborais que afetam o psicofísico e a produtividade do profissional nessas Unidades. Por se tratar de uma doença característica do meio laboral, que se dá em resposta à cronificação do estresse ocupacional trazendo consigo consequências negativas em nível individual, profissional, familiar e social.

O método utilizado foi o descritivo, com abordagem quantitativa e desenvolvido por meio de referências teóricas e pesquisa de campo com 16 profissionais que se propuseram a participar deste estudo.

Esta pesquisa realizou-se na UTI neonatal da Unidade Municipal Materno Infantil, município de Teixeira de Freitas, cidade localizada no Extremo Sul da Bahia. A UTI neonatal é composta por 10 leitos e atende toda região circunvizinha, pois o referido hospital é tido como referência em alto risco.

Como amostragem foi selecionada para participar desta pesquisa toda a equipe multiprofissional da UTI neonatal composta por 26 profissionais, sendo eles: 05 médicos, 04 enfermeiros, 04 fisioterapeutas e 13 técnicos de enfermagem. Porém, deste total, apenas 16 profissionais se disponibilizaram para a pesquisa, dentre eles 04 enfermeiros, 04 fisioterapeutas e 08 técnicos de enfermagem. Os mesmos assinaram o termo de consentimento livre, e preencheram questionários com informações pessoais e investigação do tema.

Aborda dados demográficos como: sexo, idade, estado civil, e quantidade de filhos. E características profissionais como: turno de trabalho, titulação, tempo de experiência profissional, tempo de experiência na instituição hospitalar, carga horária semanal no trabalho, numero de clientes que atente diariamente, e se trabalha exclusivamente na instituição.

$O$ instrumentos utilizados nessa pesquisa foram um questionário investigativo com fatores predisponentes pessoais de risco para a Síndrome de Burnout, juntamente com o $\mathrm{MBI}$ (Maslach Burnout Inventory) Inventário em Burnout de Maslach, com adaptação em português por Chafic Jbeili psicanalista e psicopedagogo que há mais de doze anos palestra e dá treinamentos sobre o tema para educadores.

Esse inventário contém 22 questões, sendo nove itens relacionados à exaustão emocional; cinco relacionados com a despersonalização e oito itens relacionados à baixa realização pessoal no trabalho. Cada item indica a frequência das respostas em uma escala de pontuação que vai de zero (0) a quatro (4). Sendo (0) para "nunca", (1) para "algumas vezes ao ano", (2) para "algumas vezes ao mês", (3) para indicar "algumas vezes na semana" e (4) para indicar "diariamente"(12).(SANTOS, ALVES e RODRIGUES 2009)

Os dados foram coletados no decorrer do segundo semestre do ano de 2011, após a aprovação do hospital onde os dados foram coletados. Após a confirmação e o preenchimento do termo de consentimento livre e esclarecido.

\section{RESULTADOS E DISCUSSÕES}

Dentre os 16 entrevistados, 14 (87 \%) são do sexo feminino, e 02 (13 \%) correspondem ao sexo masculino. Fogaça (2008) destaca que em Unidades de Terapia Intensiva Neonatal, o número de profissionais do sexo feminino é predominante e estas características deveriam ser levadas em conta para 


\section{Rev. Elet. em Gestão, Educação e Tecnologia Ambiental (e-ISSN: 2236-1170)}

futuros estudos, objetivando investigar o desgaste profissional encontrado em profissionais de saúde, pois um grande número das amostras estudadas em UTIPN, sobretudo na área de enfermagem são formadas por mulheres, o que poderia comprometer o estudo no que diz respeito a profissionais masculinos incluídos no mesmo entorno organizacional.

Como o número de homens não equivale a mesma porcentagem de mulheres, não é possível afirmar que estas apresentam maior predisposição para desenvolver a doença, nem obter um nível de estresse mais elevado que o sexo masculino.

A maioria dos profissionais são solteiros, correspondendo a 9 (56\%) e 7 (44\%) são casados. Um dado que estatisticamente não segue os princípios dos estudos de Millan (2007) que identificou em sua pesquisa que os casados 'sem hobby' correm maior risco de desenvolver a síndrome, já que mesmo solteiros obtiveram uma pontuação moderada em relação ao nível de estresse.

Em relação à profissão dos participantes da pesquisa, observa-se que $8(50 \%)$ deles são técnicos de enfermagem, 4 (25\%) são enfermeiros e 4 (25\%) são fisioterapeutas. Segundo Stacciarini et al. (2001) não há estudos que comprovam se há uma profissão mais estressante que outra, e sim, diferentes fatores de estresse de acordo com a função ou setor de trabalho. Embora, entende-se que o técnico de enfermagem exerce muitas funções assistenciais ao paciente por manter contato direto, lidando com a dor, sofrimento e morte.

Sobre o tempo de serviço que os profissionais da UTI neonatal exercem na mesma intituição de trabalho, percebe-se que $6(38 \%)$ trabalham neste lugar há menos de um ano, correspondendo a um nível de estresse baixo; 9 (56\%) trabalham no mesmo local entre mais de um a dez anos, podendo ter um nível de estresse maior; e, apenas $1(6 \%)$ trabalha a quase vinte anos no mesmo ambiente.

De acordo com Fernandes et al. (2008) o profissional de enfermagem tem que conviver com a ausência de horas adequadas para o repouso e também para o lazer, assim como realizar outras atividades do cotidiano, promovendo uma situação de constante estresse. è possível perceber que $12(75 \%)$ dos profissionais, sendo a maioria técnicos de enfermagem que trabalham mais de 40 horas semanais; 3 (19\%) trabalham até 40 horas semanais e apenas $1(6 \%)$ trabalha 30 horas semanais. Para Campos (2005) essa carga horária exacerbada apresentada pela maioria dos entrevistados é um fator desencadeante para a sindrome.

Dentre os entrevistados, observa-se, que 6 (37\%) praticam alguma atividade física, o que para Millan (2007) é um fator que ajuda os indivíduos a não desenvolverem a Burnout, pois ter uma atividade física ou comunitária afasta as pessoas do sedentarismo e do estresse. Porém, foi observado também que 10 (63\%) dos entrevistados não praticam nenhum exercício físico, mantendo um estilo de vida sedentária, que para o autor supramencionado é um fator de risco no desenvolvimento desta síndrome.

Segundo o portal da Cassi (2011) o médico Adriano Appel relata que o tabagismo é um vício que promove muitas doenças no corpo humano e, em muitos casos as pessoas adquirem este hábito por razões emocionais, tentando escapar de alguma situação de estresse. Percebe-se que os 16 entrevistados não fazem uso do tabaco e uso moderado de álcool.

De acordo com o Dr Rodrigo Marot (2011) não é o estresse que determina o etilismo, porém, pessoas submetidas a situações de estresse contínuo, são mais vulneráveis ao alcolismo, pois este muitas vezes é usado como ansiolítico, por ter ação relaxante e tranquilizante. Apenas 1 (6\%) entrevistado relata fazer uso frequente do álcool e 15 (94\%) deles não são adeptos ao etilismo.

Através do inventário aplicado aos participantes da pesquisa, foi possível a identificação preliminar da Burnout, onde 12 (75\%) dos entrevistados apresentaram um índice de pontuação para fase inicial da síndrome; 3 (19\%) apresentaram a possibilidade de desenvolver esta síndrome; e em 1 (6\%) entrevistado a síndrome já encontra-se instalada.

Fogaça (2008) afirma que os profissionais que trabalham em Unidade de Terapia Intensiva Pediátrica e Neonatal, pela especificidade do seu trabalho, estão expostos ao risco do estresse ocupacional e, consequentemente ao Burnout. Estes dados sugerem a necessidade de serem feitas pesquisas, com o objetivo de desenvolver medidas preventivas e modelos de intervenção. 
Rev. Elet. em Gestão, Educação e Tecnologia Ambiental (e-ISSN: 2236-1170)

\section{CONSIDERAÇÕES FINAIS}

A pesquisa foi realizada com um olhar crítico e investigativo, no qual foi analisada criteriosamente a temática em estudo: $O$ impacto do estresse ocupacional e a síndrome de Burnout na Unidade de Terapia Intensiva Neonatal.

Este estudo contou com uma pesquisa de campo que contemplou 16 profissionais da UTI neonatal do Hospital Santa Rita em Teixeira de Freitas - Bahia. Considerando as evidências da pesquisa, percebe-se que $63 \%$ dos participantes possuem algum grau de estresse, correspondendo a fase inicial da síndrome de Burnout, sendo o maior percentual, $87 \%$ do sexo feminino devido a predominância feminina na profissão de enfermagem.

Constatou-se também que entre os fatores desencadeantes para o desenvolvimento da síndrome estão: a própria função exercida e o ambiente de trabalho; a carga horária que na maioria dos casos passa de 40 horas semanais, correspondendo a 75\%; o tempo de serviço na mesma unidade que também pode influenciar o estresse ocupacional, correspondendo em sua maioria a 56\% entre 1 e 10 anos de trabalho; a ausência na prática de atividades físicas gerando o sedentarismo, no qual $63 \%$ relataram não praticar nenhum exercício.

Foi observado na pesquisa que embora o estado de estresse esteja moderado entre os entrevistados, o tabaco e o etilismo não foram usados como forma de aliviar a tensão provocada pelo trabalho.

Os estudos realizados nesta pesquisa revelam a importância de se repensar no modelo de trabalho em saúde visando a melhoria da qualidade de vida no trabalho do profissional preservando sua saúde física e mental para prestar uma assistência mais eficiente, tanto de forma técnica como humanizada aos pacientes.

Acredita-se que os objetivos propostos na pesquisa foram alcançados, corroborando a importância do conhecimento científico sobre o ambiente de trabalho do profissional intensivista.

Este estudo pretende instigar o debate acerca do estresse que é gerado no ambiente de trabalho, trazendo a discussão de uma temática pouco abordada, porém muito importante para a saúde do profissional que tem em sua essência o cuidar.

\section{REFERÊNCIAS}

Brasil. Ministério da Saúde. Formação pedagógica em educação profissional na área da saúde/enfermagem: guia do aluno. Brasília: Ministério da Saúde; 2000.

CAMPOS R. G. Burnout: uma revisão integrativa na enfermagem oncologia. Dissertação (Mestrado) Universidade de São Paulo Esc. de Enferm. de Ribeirão Preto, 2005.

CARLOTTO M. S. A Síndrome De Burnout e o Trabalho Docente Psicologia em Estudo, Maringá, v. 7, n. 1, p. 21-29, jan./jun. 2002

CASSI, Caixa de assistência dos funcionários do Banco do Brasil. Tabagismo: diminui número de fumantes no Brasil, 2011. Disponível em: http://www.cassi.com.br. Acessado em: 08/11/2011.

FERNANDES S. M. B. A.; MEDEIROS S. M.; RIBEIRO L. M. Estresse ocupacional e o medo do trabalho atual: repercussões na vida cotidiana das enfermeiras Rev. Eletr. de Enferm. 2008; 10(2): 414-427.

FISCHER, J. E.; CALAME, A.; DETTLING, A. C.; ZEIER,H.; FANCONI, S. Experience and endocrine stress responses in neonatal and pediatric critical care nurses and physicians. Critical Care Medic., v. 28, n. 9, p. 3281-3288, sept., 2000. 
Rev. Elet. em Gestão, Educação e Tecnologia Ambiental (e-ISSN: 2236-1170)

FOGAÇA, M. de C.; CARVALHO, W. B. de; CÍTERO, V. de A.; MARTINS, L. A. N. Estudo preliminar sobre a qualidade de vida de médicos e enfermeiros intensivistas pediátricos e neonatais. Rev. da Esc. de Enferm, 2010; 44(3) p.708-712.

FOGAÇA, M. de C.;CARVALHO,; CÍTERO, W. B. de; MARTINS V.A.; NOGUEIRA L. A. Fatores que tornam estressante o trabalho de médicos e enfermeiros em terapia intensiva pediátrica e neonatal: estudo de revisão bibliográfica. Rev. Bras. Ter. Intens. 2008; 20(3) p.261-266.

GAÍVA M. A. M., SCOCHI C. G. S.A participação da família no cuidado ao prematuro em UTI Neonatal Rev Bras Enferm 2005 jul-ago; 58(4):444-8.

JODAS D. A., HADDAD M. C. L. Síndrome de Burnout em trabalhadores de enfermagem de um pronto socorro de hospital universitário Acta Paul Enferm 2009;22(2):192-7

MAROT, R.. Alcoolismo: transtornos relacionados por semelhança e classificação. Disponível em: http://www.psicosite.com.br/tra/drg/alcoolismo.htm. Acessado em: 08/11/2011

MILLAN LR. A Síndrome de Burnout: realidade ou ficção? Rev. Assoc Med Bra v 53 n1 São Paulo jan-fev 2007.

OLIVEIRA P. R., TRISTÃO R. M., NEIVA E. R. Burnout e suporte organizacional em profissionais de UTINeonatal Educ. Profission.: Ciên. e Tec. Jul-Dez 2006, v1, no1, p.27-37

PAFARO R. C., MARTINO M. M. F. Estudo do estresse do enfermeiro com dupla jornada de trabalho em um hospital de oncologia pediátrica de Campinas. Rev Esc Enferm. 2004; 38(2): 152-60

SANTOS F. E., ALVES J. A., RODRIGUES A. B. Síndrome de burnout em enfermeiros atuantes em uma Unidade de Terapia Intensiva Einstein. 2009; 7(1 Pt 1):58-63

SOUZA, J. R. de. Síndrome de Burnout em enfermeiros assistencialistas no município de Teixeira de Freitas. Trabalho (Graduação) - Faculdade de Enfermagem, Pitágoras, Teixeira de Freitas - Bahia, 2010

STACCIARINI J. M. R.; TRÓCCOLI B. T. O estresse na atividade ocupacional do enfermeiro. Rev. LatinoAmeric. de Enferm. v9 no2 mar - abr 2001 Ribeirão Preto, 2001.

TOWNSEND, M. C. Enfermagem Psiquiátrica - Conceitos de cuidados. 3 edição. Rio de Janeiro - RJ. 2001 